\title{
An evaluation of machine-learning for predicting phenotype: studies in yeast, rice, and wheat
}

\author{
Nastasiya F. Grinberg ${ }^{1,2}$ (D) Oghenejokpeme I. Orhobor ${ }^{1} \cdot$ Ross D. King $^{3}$
}

Received: 28 October 2015 / Revised: 17 September 2019 / Accepted: 19 September 2019 /

Published online: 23 October 2019

(c) The Author(s) 2019

\begin{abstract}
In phenotype prediction the physical characteristics of an organism are predicted from knowledge of its genotype and environment. Such studies, often called genome-wide association studies, are of the highest societal importance, as they are of central importance to medicine, crop-breeding, etc. We investigated three phenotype prediction problems: one simple and clean (yeast), and the other two complex and real-world (rice and wheat). We compared standard machine learning methods; elastic net, ridge regression, lasso regression, random forest, gradient boosting machines (GBM), and support vector machines (SVM), with two stateof-the-art classical statistical genetics methods; genomic BLUP and a two-step sequential method based on linear regression. Additionally, using the clean yeast data, we investigated how performance varied with the complexity of the biological mechanism, the amount of observational noise, the number of examples, the amount of missing data, and the use of different data representations. We found that for almost all the phenotypes considered, standard machine learning methods outperformed the methods from classical statistical genetics. On the yeast problem, the most successful method was GBM, followed by lasso regression, and the two statistical genetics methods; with greater mechanistic complexity GBM was best, while in simpler cases lasso was superior. In the wheat and rice studies the best two methods were SVM and BLUP. The most robust method in the presence of noise, missing data, etc. was random forests. The classical statistical genetics method of genomic BLUP was found to perform well on problems where there was population structure. This suggests that standard machine learning methods need to be refined to include population structure information when this is present. We conclude that the application of machine learning methods to phenotype prediction problems holds great promise, but that determining which methods is likely to perform well on any given problem is elusive and non-trivial.
\end{abstract}

Keywords Random forest - Gradient boosting machines $\cdot$ Support vector machines $\cdot$ Lasso regression · Ridge regression · BLUP · GWAS · Statistical genetics · Plant biology

Editor: Henrik Boström.

Nastasiya F. Grinberg

ng414@cam.ac.uk

Extended author information available on the last page of the article 


\section{Introduction and background}

\subsection{Predicting phenotype}

The phenotype (physical character) of an organism is the result of interactions between the organism's complement of genes (its genotype) (Wei et al. 2014; Mackay 2014) and its environment. A central problem of genetics is to predict an organism's phenotype from knowledge of its genotype and environment. This problem is now of the highest societal importance. For example, human disease is a phenotype, and understanding its relation to genotype and environment is a central problem in medicine (Stranger et al. 2011; Lee et al. 2011), see for example the studies in schizophrenia (Schizophrenia Working Group of the Psychiatric Genomics Consortium 2014), obesity (Locke et al. 2015), etc. Similarly, crop yield and drought resistance are phenotypes, and if we are going to be able to continue to feed the world's growing population, it is essential to better predict crop phenotypes from knowledge of their genotypes and environment (Buckler et al. 2009; Jannink et al. 2010; Hayes and Goddard 2010; Brachi et al. 2011; Desta and Ortiz 2014). For such reasons, the problem of predicting phenotype has recently (2016) been listed by the US National Science Foundation as one of its six key 'Research Frontiers': 'understanding the rules of life'.

Our ability to predict phenotype is being revolutionised by advances in DNA sequencing technology. These advances have enabled, for the first time, an organism's genotype to be extensively characterised-typically via thousands of genetic markers. The cost of sequencing is decreasing rapidly, which means that it is now often low enough that in a single investigation many organisms (hundreds/thousands) may be genotyped, which opens up the possibility of using statistics/machine learning to learn predictive relationships between an organism's genotype, environment, and phenotype. Such studies are often called genomewide association studies (GWAS).

The traditional focus of most GWAS has been on the discovery of genetic markers (normally only a small number) that are 'associated' (i.e., correlated) with a phenotype. However, rather than being true causal mutations, called quantitative trait loci (QTL), these markers are usually correlated to QTLs and serve as proxies. Such single-nucleotide polymorphisms (SNPs) and QTLs are usually in linkage disequilibrium (LD), i.e. within a given population, their alleles are correlated more than would be expected by chance. In GWAS, focusing on a small number of genes has significant biological limitations, as most biological phenotypes result from the interaction of multiple genes and the environment; and the focus on association rather than prediction has statistical limitations, as it makes the objective evaluation of the utility of results difficult.

The current trend is therefore towards a more direct and operational approach to phenotype prediction problems: learn a predictive function that, from the input of an organism's genotype and environment, predicts its phenotype (see, for example, Yang et al. 2010; Bloom et al. 2013; Desta and Ortiz 2014; Shigemizu et al. 2014). Special purpose statistical genetics methods have been developed for this task (see, for example, Lynch and Walsh 1998; Bloom et al. 2013; Desta and Ortiz 2014). Predictive phenotype problems are also clearly well suited for standard machine learning methods.

In this paper, we compare for phenotype prediction a state-of-the-art classical statistical genetics method and a mixed-model approach BLUP (used extensively in genomic selection applications) with standard machine learning methods. We investigate how these methods perform on three very different types of phenotype prediction problem, one from yeast Saccharomyces cerevisiae (Bloom et al. 2013), the other two from wheat Triticum aestivum L. 
(Poland et al. 2012) and rice Oryza sativa (Alexandrov et al. 2015). We also compare how performance varies with the complexity of the biological mechanism, the amount of observational noise, the number of examples, the amount of missing data, and the use of different data representations.

\subsection{Phenotype prediction data}

Genomic data has a specific structure that strongly influences the application of machine learning methods. We therefore first briefly describe this structure using standard machine learning terminology—rather than the terminology of statistical genetics which may confuse the uninitiated.

Representing genotype. The complete genotype of an organism consists of the linear arrangement of its genes on chromosomes, together with the sequences of all the genes and intergenic regions. Genotype information is normally represented in phenotype prediction problems as 'markers', these are discrete attributes that signify that a particular stretch of DNA varies between organisms. Usually, these variations are mutations at a single position (base pair) in a DNA sequence called SNPs. As organisms of the same species mostly share the same DNA sequences, this representation is reasonably concise, but as genomes are large, many thousands of markers are typically needed to characterise an organism's genome. (It should be noted that this propositional marker representation is sub-optimal, as it ignores a substantial amount of information: the biological context of the markers involved, the linear ordering of the markers, etc.) In this paper, we utilise this marker representation as it is standard, and it simplifies the application of standard statistical/machine learning methods.

In phenotype prediction problems, it is preferable for all the organisms (examples) to have their genotypes fully sequenced, as this provides the maximum amount of information. However, this is not possible in many problems, either because of technical reasons or cost. In such cases, the genotypes are not fully characterised. In this paper we investigate two problems (yeast and rice) where all the organisms are fully sequenced, and another (wheat) where only a subset of markers is known, and the organism's genome has not yet been sequenced because of its complexity.

Environment. Prediction of phenotype is easier if the environment is controlled. However, this is difficult or impossible to do in many cases, for example, in studies involving humans many aspects of the environment are unknown, in outdoor crop studies the weather cannot be controlled, etc. In this paper, we investigate one problem (yeast) where the environment is fully controlled (well-defined laboratory conditions), and another two (wheat and rice) where the environment is partially controlled.

Measuring phenotype. Due to the continuing steep fall in DNA sequencing costs in many phenotype prediction problems, the most expensive step is the observation of an organism's phenotype. This means that in many scenarios the number of attributes is greater than the number of examples. Furthermore, it has also led to new genetic methodologies based on phenotype prediction, an example of which is genomic selection (Meuwissen et al. 2001; Heffner et al. 2009). In this paper, we investigate one problem (yeast) where the observation of phenotype (growth) is cheap as it involves laboratory automation equipment, and another (wheat and rice) where it is expensive and time-consuming-it takes many months for a harvest.

Causation of phenotype. The number of genetic mutations involved in causing a phenotype can vary greatly between phenotypes. For example, the phenotype of pea colour (yellow or green), that was classically studied by Gregor Mendel, is caused by variations (polymor- 
phisms) in a single gene (stay-green) (Armstead et al. 2007). Therefore, given knowledge of markers in stay-green, one can usually very accurately predict pea colour. In contrast, the phenotype of human height (classically studied by Sir Francis Galton in the original 'regression' problem) involves a large number of genes and environmental effects - the central-limit theorem thereby explains why human height is roughly normally distributed when controlled for sex and geographical population (Wood et al. 2014).

An important feature of genetic data is that the examples (organisms) are formed by meiosis (sex), where parents shuffle their genomes when forming offspring. The mechanisms involved in meiosis are complicated, but, the result is that each of the child cell's chromosomes consists of a random patchwork of linear sections taken from the two parents (Fig. 1a). This means that if two markers are close together on an organism's DNA, then the associated mutations (allele types) are likely to be inherited together. Consequently, attributes (markers) can be highly correlated (linkage disequilibrium). It also means that all the markers in each of these linear sections are identical to that of one parent so that the variety of children that are likely to be formed is constrained.

Due to meiosis populations of organisms typically have complex ancestral (pedigree) interrelationships. These interrelationships take the form of a directed acyclic graph (DAG), with meiosis events being the nodes. However, in many phenotype prediction problems, the structure of the DAG is unknown. (Note that the DAG imposes a natural metric between examples (organisms) based on genetic changes resulting from meiosis.) Much of classical statistical genetics research has focused on dealing with population structure. For example, the BLUP (best linear unbiased prediction) method encodes genetic similarities between pairs of individuals in a genomic similarity matrix (GSM) (Meuwissen et al. 2001; Speed and Balding 2014).

Many organisms have multiple genomes in each cell. Humans have single copies (haploid) in their sex cells (sperm/eggs), but otherwise have two copies (diploid), one from their father and the other from their mother. This complicates phenotype prediction as it is not clear how the genomes interact to cause phenotype. This complication is related to multiinstance learning problems (Ray and Page 2001). In this paper, we investigate one problem (yeast) where the observed organisms are haploid, i.e., there is no complication with multiple genomes. Rice is diploid, with two paired sets of genomes, while wheat is hexaploid with three pairs of paired genomes.

\subsection{Types of phenotype prediction problem}

The simplest form of phenotype prediction problem is the case when a pair of parent organisms breed to produce a large set of offspring. In such cases the offspring can reasonably be assumed to be randomly generated from a given distribution: indeed the analogy with randomly dealing hands of cards is close and commonly used in genetics (Hogben 1946). This type of phenotype prediction problem is closely connected to practical phenotype prediction problems, for example: which embryo to select?

To investigate this type of problem, we utilised a large phenotype prediction study in yeast (Bloom et al. 2013). In this study there are a large number of examples, the complete genomes of the organisms are known, the organisms are haploid, a large number of different environments were investigated under controlled laboratory conditions, and the phenotype of growth was accurately measured. Taken together, these features give a phenotype predictions dataset that is as clean and complete as it is currently possible to get. Moreover, the uniform 
laboratory conditions under which the yeast was grown ensured that there were no (or nearly no) confounding environmental factors.

We chose as a second, and comparative, phenotype prediction problem-the real-world problem of predicting phenotype in crops: wheat (Poland et al. 2012) and rice (Alexandrov et al. 2015). This type of phenotype prediction problem is typical of genomic selection problems in organisms, in which genome-wide molecular markers are used to predict the breeding utility of individuals or breeding lines prior to phenotyping. The wheat dataset we investigated comes from a study involving 254 varieties (breeding lines) of wheat (Poland et al. 2012). These varieties were derived through six generations of meiosis (crosses) from a set of ancestor varieties. Experimental design methods were used to control the environment, and different irrigation techniques investigated. This dataset is more complex and difficult to predict than the yeast one for a number of reasons: the complete genotypes of the organisms are not known (only the markers, indeed wheat has still not been fully sequenced), the organisms are hexaploid, the organisms come from different parents (although there is some knowledge of the relationships between parents), there are fewer examples, and the environment is not fully controlled.

The rice dataset comes from the 3000 rice genomes project (Alexandrov et al. 2015). Like the wheat dataset, this problem also involves the selection of individuals that will serve as parents for the next generation of progeny using genomic predictions. The phenotype data is from different years of screening without replication. However, the values do not show significant variation due to environmental differences, as the data is part of a routine characterization of genetic resources performed by the International Rice Genebank at the International Rice Research Institute.

\subsection{Classical statistical-genetics methods for predicting phenotype}

The most common classical statistical genetics approach to the analysis of genotype, environment and phenotype data has been to use univariate and bivariate statistical methods (Lynch and Walsh 1998; Westfall et al. 2002; Marchini et al. 2005). These typically test each marker (or pairs of markers) for association with a phenotype individually and independently of the other markers. The focus on such approaches seems to have been because of a desire to understand, and possibly control, mechanisms that causes phenotype, through identification of the markers involved. This is reasonable, but the assumption of independence does not reflect the complex causal relationships involved in phenotype formation (e.g., it ignores systems biology), and it is prone to missing markers with small effects.

The emphasis on univariate and bivariate correlations also raises the problem of multiple testing and is hindered by typical $p$-value limitations, such as dependence on sample size, minor allele frequency, and difficulty to determine a meaningful threshold for the study. The multiple testing problem may be classically addressed by using false discovery rate (FDR) (Benjamini and Hochberg 1995) instead of the conventional $p$-values or the Bonferroni correction. One of the more recent approaches is to use Bayes factors instead of $p$-values (Wakefield 2007), thus taking prior belief of association into account. The problem of the interdependence of hypotheses in multiple testing (that is, possible interactions between markers) has been addressed for example by using hidden Markov models (Sun and Tony Cai 2009) and graphical models (Liu et al. 2012). In statistical genetics, arguments based on multiple testing are often used to claim that it is not possible to identify complicated interactions between markers in datasets that are not very large (Gauderman 2002; Wang and 
Zhao 2003). These arguments are incorrect as they would imply that multivariate learning is generally very difficult, which is not the case.

The emphasis in classical statistical genetics on univariate and bivariate methods research has also led to efforts to reduce the dimensionality of GWAS problems. This, for example, can be done by grouping markers in haplotypes-specific arrangements of alleles from a parent (Clark 2004). This enables the simultaneous testing of associations between a phenotype and several markers in a target region. However, identifying meaningful haplotypes is in itself a non-trivial task (Meng et al. 2003; Lin and Altman 2004).

The recent change of focus from association to prediction cuts through most of the problems of the significance of associations: the validity of a multivariate relationship between markers is demonstrated by success of predictions on test data. The utility of using test data is appreciated in statistical genetics (Lynch and Walsh 1998; Bloom et al. 2013; Desta and Ortiz 2014; Shigemizu et al. 2014) but should perhaps be stressed more. In addition, the importance of considering all the markers simultaneously has now been widely recognised, e.g. de los Campos et al. (2013), with various multivariate linear models gaining popularity. Amongst them are genomic BLUP (Meuwissen et al. 2001; VanRaden 2008) (a mixed-model related to ridge regression with a pre-specified penalty parameter) and other penalised regression methods (Gianola et al. 2006; De Los Campos et al. 2009; Li and Sillanpää 2012) and Bayesian techniques (Meuwissen et al. 2001; De Los Campos et al. 2009; Habier et al. 2011; Guan and Stephens 2011; Zhou et al. 2013). An important extension of BLUP proposed by Speed and Balding (2014) relaxes the assumption of constant variance for SNP effects. Many improved and efficient linear mixed model (LMM) algorithms have also been introduced in recent years (Kang et al. 2008; Zhang et al. 2010; Lippert et al. 2011; Loh et al. 2015; Lee and van der Werf 2016), some of which are capable of dealing with several correlated phenotypic traits (Korte et al. 2012; Zhou and Stephens 2014; Casale et al. 2015) (see also Widmer et al. 2014 and references therein). The attractiveness of these linear techniques lies in the fact that they take population structure and genetic relatedness into account (Price et al. 2010). However, most of these techniques have difficulty accounting for interactions.

\subsection{The applicability of machine learning methods}

Standard off-the-shelf machine learning methods (Dudoit et al. 2002; Ziegler et al. 2007; Szymczak et al. 2009; Ogutu et al. 2011, 2012; Pirooznia et al. 2012; Mittag et al. 2012; Okser et al. 2014; Leung et al. 2016; Cherlin et al. 2018) present an attractive alternative to classical statistical genetics methods. These methods are easy to use, are freely available in a variety of implementations, and intrinsically multivariate. In addition, machine learning methods are available that perform attribute selection, e.g., lasso and regression trees, and there are also machine learning methods available that can identify complex interactions between attributes (e.g., random forest, gradient boosting machines, neural nets), not simply bivariate ones. In a typical GWAS set-up, there is the $p \gg n$ problem, where the number of attributes $(p)$ greatly exceeds the number of sample points $(n)$, and this is a problem for classical multivariate regression. This is less of a technical problem for some machine learning methods, but of course, basic information-theoretic results imply that the underlying signal needs to be strong enough for machine learning methods to work.

One important difference between traditional statistical genetic methods and standard machine learning methods is that the machine learning methods do not require assumptions to be made about the genetic mechanism underlying a trait in question, e.g., additivity of effects, the number and size of interactions, depth of interactions, etc. This is both a strength 
and a weakness. The lack of need for these assumption simplifies the use of the methods. However, if this information is known then it should be used to aid prediction (Jaynes 2003). It should be noted that standard machine learning methods are not assumption-free. All learning methods require some inductive bias (Mitchell 1997). However, the assumptions of standard machine learning methods are more general than those of traditional statistical genetic methods.

We note that attribute selection is a potentially harder problem than prediction. In particular, markers found by GWAS (by whatever technique) to be associated to a particular trait are associated with that trait—-they are not necessarily causal but are rather in linkage disequilibrium with the true causal QTL(s). Finding causal genetic variants is the subject of statistical fine-mapping (Schaid et al. 2018) and is beyond the scope of this paper. More generally, the ultimate goal is to build Systems Biology models of biological systems that faithfully reflect the underlying biology, and which can be used for mechanistic predictions. In this work, we concentrate on finding good machine learning predictive models and make no pretense of learning causal mechanistic models.

\section{Materials and methods}

\subsection{Experimental data}

The yeast dataset was derived from a study of 1008 haploid yeast strains derived from a cross (meiosis) between a laboratory and a wine strain of the yeast Saccharomyces cerevisiae. The parent strains differed by $0.5 \%$ at the sequence level. The genotypes of the parents and children were determined by sequencing (Fig. 1). The raw sequence data was transformed into 11,623 Boolean markers: coded to be ' 1 ' if the sequence variation came from the wine strain (RM) parent and ' 0 ' if it came from the laboratory strain (BY) parent.

The environment of the yeast strains was modified in 46 different ways (Table 1): the basic chemicals used for growth were varied (e.g., galactose, maltose), minerals added (e.g., copper, magnesium chloride), herbicides added (paraquat), etc.

Yeast population growth (growth is the most basic of all phenotypes) was measured under these different conditions. As the data was generated by high-throughput robotics there are many missing values; there are, for example, only 599 readings available for sorbitol. Most traits, however, have upwards of 900 readings, some with two replications (which we average). All the growth measurements are normalised to have a mean of 0 and a variance of 1.0.

Using this yeast study, we investigate different aspects of applying machine learning to phenotype prediction data by starting with as clean data as possible, and then gradually artificially degrading it to make it resemble different practical phenotype prediction problems in animals and plants. A complementary motivation for using such clean and complete data is that with improved technology applied phenotype prediction problems will increasingly resemble this clean, comprehensive form.

The wheat dataset comes from a genomic selection study in wheat involving 254 breeding lines (samples) with genotypes represented by 33,516 SNP markers coded as $\{-1,0,1\}$ to correspond to the $a a, a A$ and $A A$ alleles, respectively (Poland et al. 2012). Missing values were imputed with heterozygotes $a A$ (the original paper found little difference between four different imputation methods, one of which was imputing with heterozygotes). The wheat lines were evaluated in plots for four phenotypic traits with three replicates (which were averaged): yield (drought), yield (irrigated), thousand kernel weight (TKW) and days to 


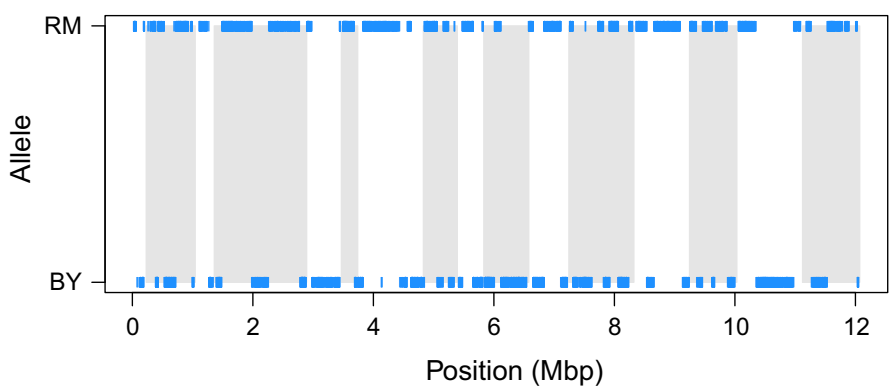

(a)

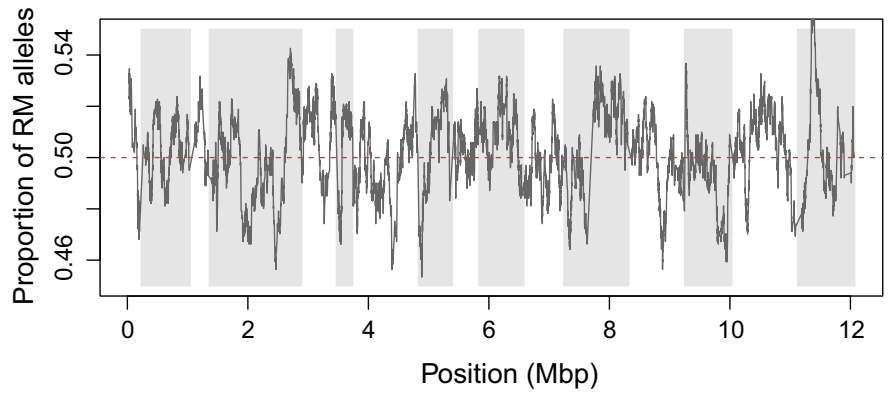

(b)

Fig. 1 a Markers' expression for a RM $\times$ BY yeast strain [segregant A01-01 (Bloom et al. 2013)] plotted against their position (mega base pairs) on the genome. Each tick represents a marker, bands represent chromosomes. 1/0 marker values correspond to RM/BY parent, respectively. b Proportion of markers coming from the RM parent plotted against markers position (mega base pairs) on the genome. White and grey bands separate the 16 chromosomes

heading (number of days before a grass plant shows flowers or seed heads in spring; DTH). Phenotypic values were once again normalised.

For the rice data, the Core SNP subset of 3000 Rice Genomes version 0.4 from SNPSEEK (Mansueto et al. 2016) was used. The genotypes for the samples in the dataset originally contained 996,009 SNP markers. However, a subset of 101,595 markers was used in this study to reduce computational complexity. These markers were selected by linkage disequilibrium in Plink (Purcell et al. 2007), using the -indep-pairwise command with a window of 50 SNPs, a step size of 5 , and $r^{2}$ value of 0.02 . The markers were coded in the same way as in the wheat dataset, and missing values were imputed using column means as is common in the rice literature (Spindel et al. 2015). We note that this imputation method effectively results in some information exchange between training and test portions of the data but in practice this should not have a significant effect on results. Indeed, it has been shown that mean imputation is sufficient in cases where less than $20 \%$ of the data for each marker is missing (Rutkoski et al. 2013), which is the case for our dataset. Twelve phenotypic traits were considered: culm diameter, culm length, culm number, grain length, grain width, grain weight, days to heading, ligule length, leaf length, leaf width, panicle length, and seedling height. Due to missing phenotype data, each trait has its own set of samples, with the number of samples ranging from 1877 to 2265 .

In statistical/machine learning terms: each of the different genotype/phenotype combinations represents a different regression problem. The yeast strains/wheat/rice samples are the 

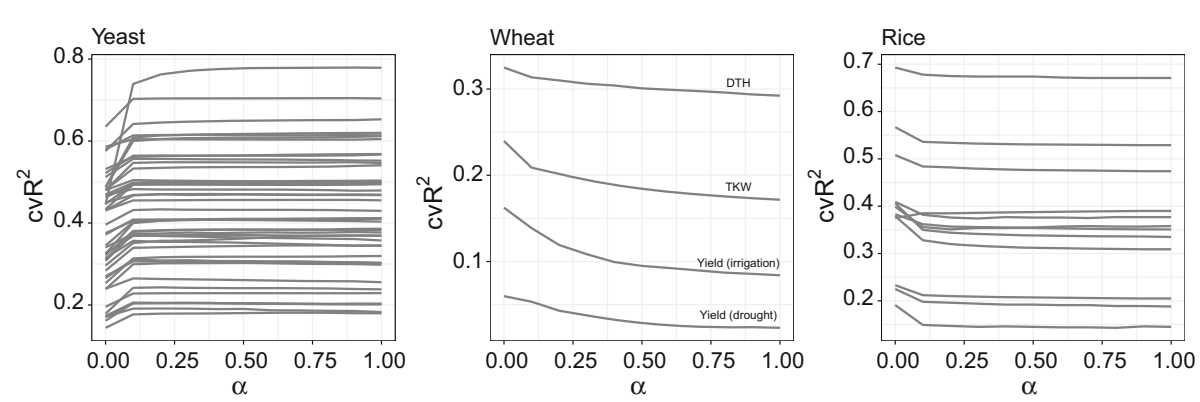

Fig. 2 Comparison of performance of elastic net on yeast, wheat and rice datasets for varying values of the $\alpha$ parameter for each trait with $\alpha$ on the $x$-axis and cv $R^{2}$ on the $y$-axis

examples, the markers in the examples are the attributes, and the growth of the strains (for yeast) and agronomic traits evaluated (for wheat and rice) are variables to be predicted.

\section{Learning methods}

\subsection{Standard statistical and machine learning methods}

We investigated several variants of penalised linear regression: elastic net (Zou and Hastie 2005), ridge regression (Hoerl and Kennard 1970), and lasso regression (Tibshirani 1996). The rationale for choosing these methods is that they most closely resemble the multivariate approaches used in classical statistical genetics. In genomic applications, we expect a lot of highly correlated variables (SNPs in LD). Ridge regression tends to shrink coefficients of highly correlated attributes towards each other, while lasso regression chooses between highly correlated strong variables, albeit in an ad hoc fashion. Neither approach is ideal, although lasso's ability to produce a sparse solution can be a useful feature. The advantage of both these methods is the simplicity of the resulting linear model. We also investigated an array of models that interpolate between ridge and lasso regression through use of an elastic net penalty. We considered 11 values of the elastic net penalty $\alpha$ evenly spaced between 0 (ridge) and 1 (lasso) with the value of the overall penalty parameter $\lambda$ chosen by cross-validation (CV) separately for each value of $\alpha$ (see Fig. 2).

We also investigated the tree methods of random forests (Breiman 2001) and gradient boosting machines (GBM) (Friedman 2001). The rationale for the use of these is that they are known to work robustly, and have an inbuilt way of assessing the importance of attributes. Both of these methods work by combining a multitude of individual regression trees. Hence at an individual tree level, any interactions between attributes should be accounted for. However, uncovering these interactions is not easy, and is an area of ongoing research (see e.g. Wright et al. 2016; Li et al. 2016; Lampa et al. 2014). Additionally, unlike the above mentioned linear methods, RF, and especially GBM, can require careful tuning for optimal performance. For random forests, we used 700 iterations for yeast and wheat and 1000 iterations for rice (chosen to be enough for convergence) of fully-grown trees with the recommended values of $p / 3$ (where $p$ is the number of attributes) for the number of splitting variables considered at each node, and 5 examples as the minimum node size. For GBM we tuned two parameters via internal train/test split inside each fold: interaction depth and shrinkage. We investigated interaction depths of 1, 2 and 3 and shrinkage of $0.001,0.01$ and 0.1 (in our experience 


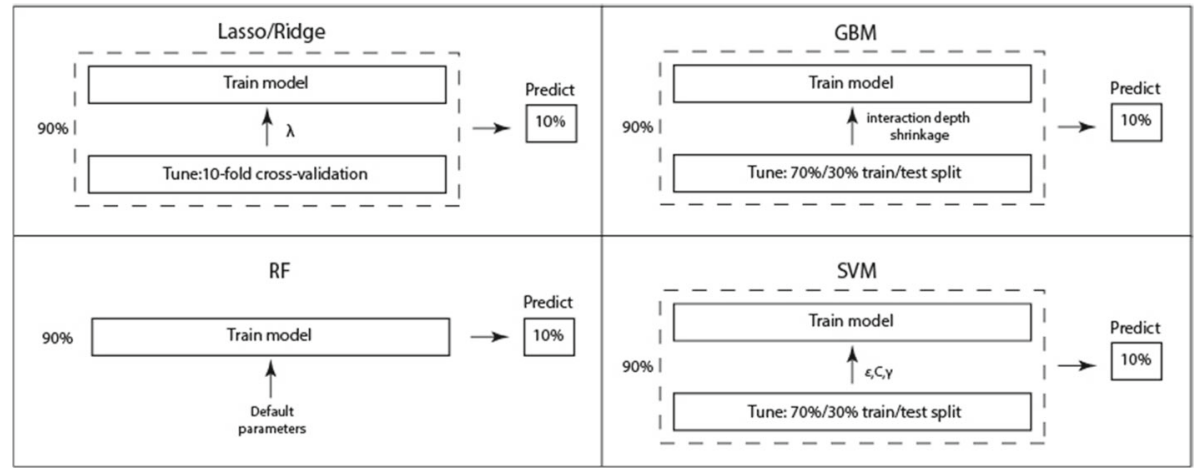

Fig. 3 Tuning, training and testing the five ML methods within the cross-validation framework (note that the above diagram is for the tenfold $\mathrm{CV}$ performed on the yeast dataset; for fivefold CV performed on the wheat dataset training and test folds above should be $80 \%$ and $20 \%$, respectively)

the default shrinkage parameter of 0.001 lead to too slow of a convergence), resulting in a two-dimensional parameter grid. We used 1000 trees, which was enough for convergence for all traits. The optimal number of iterations for predictions was determined via an assessment on an internal validation set within each cross-validation fold. Finally, we used the default value of 0.5 as the subsampling rate.

Finally, we investigated support vector machines (SVM) (Cortes and Vapnik 1995). SVM methods have been gaining popularity in phenotype prediction problem recently. However, experience has shown that they need extensive tuning (which is unfortunately extremely time-consuming) to perform well (Hsu et al. 2008). We used $\epsilon$-insensitive regression with Gaussian kernel and tuned the model via internal testing within each cross-validation fold over a fine grid (on the logarithmic scale) of three parameters: $\epsilon$, cost parameter $C$ and $\gamma$ (equal to $1 /\left(2 \sigma^{2}\right)$, where $\sigma^{2}$ is the Gaussian variance).

For yeast (tenfold CV) and wheat datasets (fivefold CV), during each CV iteration parameter tuning is performed within the training fold comprising $90 \%$ (or $80 \%$ ) of the data. For GBM and SVM, we tune and validate parameters on the $70 \%$ and $30 \%$ of the training fold, respectively. For lasso, tuning of $\lambda$ is done through tenfold $\mathrm{CV}$ within the training fold. We then use the whole training fold to fit the final model using optimal parameters. Finally, we use this model to predict the held out $10 \%$ (or 20\%) of the data (test fold): this is our CVprediction for this iteration. We thus accumulate $\mathrm{CV}$ predictions over the $10 \mathrm{CV}$ iterations to be used later to assess each model's performance (see Fig. 3).

All analysis was performed in R ( $\mathrm{R}$ Core Team 2018) using the following packages: GLMNET for elastic net, RANDOMFOREST for random forest, GBM for gradient boosting and KERNLAB for support vector machines. The CARET package was used for tuning GBM and SVM.

\subsection{Classical statistical genetics}

To compare the machine learning methods with state-of-the-art classical genetics prediction methods we re-implemented the prediction method described in the original yeast Nature paper (Bloom et al. 2013), and applied the genomic BLUP model. The 'Bloom' method has two steps. In the first, additive attributes are identified for each trait in a four-stage iterative procedure, where at each stage only markers with LOD significance at $5 \%$ false 
discovery rate (identified via permutation tests) are kept and added to a linear model; residuals from this model are then used to identify more attributes in the next iteration. In the second step, the genome is scanned for pairwise marker interactions involving markers with significant additive effect from the previous step by considering likelihood ratio of a model with an interaction term to a model without such a term. We reapplied the first step of the analysis to the yeast dataset using the same folds we used for cross-validation for our ML methods. Additionally, we altered the CV procedure reported in the Nature paper (Bloom et al. 2013) as it was incorrect (the authors incorrectly identified QTLs on the training fold, but both fitted the model and obtained predictions on the test fold, which unfortunately overestimates the obtained $R^{2}$ values, one of the pitfalls described by Wray et al. (2013)). We selected attributes and constructed the models only using the data in a training fold, with predictions obtained by applying the resulting model to the test fold.

The genomic BLUP model is a linear mixed-model similar to ridge regression but with a fixed, biologically meaningful penalty parameter. BLUP takes relatedness of the individuals in the study into account via a genetic relatedness matrix computed from the genotypic matrix. The reason for choosing this method is that it (and its various extensions) is a very popular approach in genomic selection, and was the method applied in the original wheat paper (Poland et al. 2012). We used the R implementation in the RRBLUP package (Endelman 2011).

\subsection{Evaluation}

The performance of all models was assessed using tenfold and fivefold cross-validation, for yeast and wheat, respectively and a train/test split for rice. Cross-validated predictions were collected across the folds and then used to calculate $R^{2}$ (informally-proportion of variance explained by the model) in the usual manner (we call this measure cross-validated $R^{2}-\mathrm{cv} R^{2}$ ). The wheat dataset was small enough to repeat the $\mathrm{CV}$ procedure several times: we accumulated $\mathrm{cv} R^{2}$ across 10 runs on different fold selections and reported the average values. Formally, $\mathrm{cv} R^{2}$ is defined as:

$$
\mathrm{cv} R^{2}=1-\frac{\sum_{i}\left(\tilde{y}_{i}-\bar{y}\right)^{2}}{\sum_{i}\left(y_{i}-\bar{y}\right)^{2}},
$$

where $\left(y_{1}, \ldots, y_{n}\right)$ is the vector of outputs, $\left(\tilde{y}_{1}, \ldots, \tilde{y}_{n}\right)$ is the vector of cross-validated predictions (see Fig. 3), and $\bar{y}$ is the sample mean of outputs.

For rice, models were trained on $70 \%$ of the data and performance assessed on the remaining $30 \%$.

Next, we used the Friedman test to check whether performance rankings of various methods were significantly different from each other. For each dataset, the Friedman test proceeds by first ranking performances of the methods separately for each trait. Average rank across all traits is then calculated for each method and a null-hypothesis that all the average ranks are equal, i.e. that all the methods are equivalent, is formally tested (see Demšar (2006)). Finally, a post-hoc Nemenyi test was applied to assess differences between individual pairs of methods. 


\section{Results}

\subsection{Overall comparison of methods}

Tables 1, 2 and 3 summarise the cv $R^{2}$ and $R^{2}$ values for the standard statistical/machine learning methods, the Bloom GWAS method and BLUP for yeast, wheat and rice, respectively. The elastic net results are represented by the extremes, lasso, and ridge, as predictive accuracy appears to be a monotonic function of the elastic net penalty parameter $\alpha$ for all the datasets (see Fig. 2). Moreover, elastic net tends to side with the strongest method (lasso for yeast and ridge for wheat and rice). This might bring justification for just using the $\alpha=0.5$ mixture parameter (as is done in some literature, e.g. Gamazon et al. (2015); Cherlin et al. (2018)) or simply trying the two extremes instead of the comprehensive tuning of $\alpha$.

The yeast results show that there is at least one standard machine learning approach that outperforms Bloom and BLUP on all but 6 and 5 traits, respectively. In addition, the mean advantage of the Bloom (BLUP) method on these 6 (5) traits is marginal: $1.8 \%(0.4 \%)$, with a maximum of $4.1 \%(1.5 \%)$, whilst the mean advantage of the standard machine learning techniques is $5.0 \%(5.5 \%)$, with a maximum of $14.2 \%(27.5 \%)$-for SVM (GBM) on tunicamycin (maltose). (N.B. we did not re-run the second stage of the Bloom's procedure, mining for pairwise marker interactions, but used the paper's original results, so the actual cv $R^{2}$ results for traits with interactions for Bloom's method should be slightly lower than in Table 1). Across the machine learning methods, the best performing method was GBM, which performed best in 26 problems. For 6 problems each lasso and the method of Bloom won. BLUP and SVM showed the best results for 5 and 3 traits, respectively. SVM on the whole across traits performed very similarly to BLUP but required time-consuming tuning.

At $p$-value of $2.2 \times 10^{-16}$, the Friedman test indicates a highly significant difference between performances of various methods. Furthermore, pairwise post-hoc Nemenyi tests indicate that GBM is significantly different from Bloom and BLUP methods, at $p$-values of $3.4 \times 10^{-8}$ and $9.3 \times 10^{-5}$, respectively.

The results for the wheat dataset paint quite a different picture: SVM performs the best for all traits (albeit with a marginal advantage for 3 out of 4 traits), followed closely by genomic BLUP. Both of the tree methods underperform compared to SVM and BLUP. The weakest method overall is lasso. The Friedman test returns a $p$-value of 0.0015 . The only two significant pairwise comparisons are lasso/BLUP and lasso/SVM ( $p$-values of 0.03 and 0.002 , respectively), so there is no significant difference between the two closest performing methods, SVM and BLUP.

On the rice dataset, SVM performs best for 6 of the 12 traits, genomic BLUP outperforms all other methods on 4 of the 12, and on a trait each, lasso and ridge perform best (see Table 3 ). As with the wheat dataset, the tree methods are also outperformed by BLUP and SVM, and the weakest method overall is also lasso. We hypothesise that BLUP's ability to take genetic relatedness of the individuals into account gives it an advantage over the other two penalized regressions and also the two tree models. Once again, the Friedman test is significant with a $p$-value of $1.54 \times 10^{-5}$, and BLUP and SVM, the two best performing methods, differ significantly from the three ML methods of GBM, RF and lasso in pairwise comparisons.

The rest of this section is devoted to studying the performance of the five ML methods and BLUP on the yeast dataset in greater detail. As noted above, this form of a dataset is arguably the cleanest and simplest possible. 
Table $1 \mathrm{cv} R^{2}$ for the five ML methods, BLUP and for the QTL mining approach of Bloom et al. applied to the yeast dataset. The best performance for each trait is in boldface. The average ranks for computation of the Friedman test are on the bottom line

\begin{tabular}{|c|c|c|c|c|c|c|c|}
\hline Trait/method & Bloom et al & Lasso & Ridge & BLUP & GBM & $\mathrm{RF}$ & SVM \\
\hline Cadmium chloride & 0.780 & 0.779 & 0.445 & 0.556 & 0.797 & 0.786 & 0.565 \\
\hline Caffeine & 0.197 & 0.203 & 0.171 & 0.229 & 0.250 & 0.236 & 0.234 \\
\hline Calcium chloride & 0.198 & 0.255 & 0.240 & 0.268 & 0.261 & 0.205 & 0.261 \\
\hline Cisplatin & 0.297 & 0.319 & 0.253 & 0.290 & 0.338 & 0.275 & 0.272 \\
\hline Cobalt chloride & 0.431 & 0.455 & 0.431 & 0.457 & 0.460 & 0.398 & 0.448 \\
\hline Congo red & 0.460 & 0.504 & 0.469 & 0.500 & 0.500 & 0.398 & 0.487 \\
\hline Copper & 0.405 & 0.345 & 0.284 & 0.331 & 0.456 & 0.406 & 0.338 \\
\hline Cycloheximide & 0.466 & 0.498 & 0.480 & 0.516 & 0.513 & 0.444 & 0.529 \\
\hline Diamide & 0.417 & 0.479 & 0.468 & 0.498 & 0.460 & 0.318 & 0.486 \\
\hline E6_Berbamine & 0.380 & 0.403 & 0.372 & 0.399 & 0.412 & 0.281 & 0.390 \\
\hline Ethanol & 0.486 & 0.495 & 0.434 & 0.460 & 0.518 & 0.475 & 0.455 \\
\hline Formamide & 0.310 & 0.238 & 0.179 & 0.232 & 0.350 & 0.298 & 0.240 \\
\hline Galactose & 0.201 & 0.183 & 0.171 & 0.211 & 0.235 & 0.219 & 0.217 \\
\hline Hydrogen peroxide & 0.362 & 0.377 & 0.308 & 0.365 & 0.385 & 0.355 & 0.399 \\
\hline Hydroquinone & 0.135 & 0.201 & 0.173 & 0.225 & 0.212 & 0.191 & 0.188 \\
\hline Hydroxyurea & 0.232 & 0.303 & 0.266 & 0.303 & 0.336 & 0.243 & 0.320 \\
\hline Indoleacetic acid & 0.480 & 0.302 & 0.239 & 0.301 & 0.476 & 0.458 & 0.310 \\
\hline Lactate & 0.523 & 0.568 & 0.522 & 0.552 & 0.561 & 0.510 & 0.557 \\
\hline Lactose & 0.536 & 0.567 & 0.532 & 0.562 & 0.582 & 0.530 & 0.565 \\
\hline Lithium chloride & 0.642 & 0.704 & 0.635 & 0.670 & 0.711 & 0.538 & 0.680 \\
\hline Magnesium chloride & 0.278 & 0.229 & 0.196 & 0.250 & 0.266 & 0.255 & 0.267 \\
\hline Magnesium sulfate & 0.519 & 0.369 & 0.326 & 0.360 & 0.492 & 0.434 & 0.378 \\
\hline Maltose & 0.780 & 0.620 & 0.488 & 0.534 & 0.809 & 0.806 & 0.522 \\
\hline Mannose & 0.230 & 0.202 & 0.162 & 0.210 & 0.255 & 0.234 & 0.215 \\
\hline Menadione & 0.388 & 0.412 & 0.375 & 0.407 & 0.432 & 0.396 & 0.402 \\
\hline Neomycin & 0.556 & 0.614 & 0.580 & 0.609 & 0.600 & 0.487 & 0.597 \\
\hline Paraquat & 0.388 & 0.496 & 0.447 & 0.474 & 0.488 & 0.298 & 0.479 \\
\hline Raffinose & 0.317 & 0.357 & 0.341 & 0.371 & 0.383 & 0.368 & 0.364 \\
\hline SDS & 0.348 & 0.411 & 0.345 & 0.386 & 0.393 & 0.337 & 0.383 \\
\hline Sorbitol & 0.424 & 0.369 & 0.296 & 0.333 & 0.379 & 0.383 & 0.318 \\
\hline Trehalose & 0.489 & 0.500 & 0.463 & 0.487 & 0.515 & 0.472 & 0.477 \\
\hline Tunicamycin & 0.492 & 0.605 & 0.586 & 0.618 & 0.618 & 0.385 & 0.634 \\
\hline x4-Hydroxybenzaldehyde & 0.442 & 0.411 & 0.325 & 0.365 & 0.471 & 0.404 & 0.355 \\
\hline $\mathrm{x} 4 \mathrm{NQO}$ & 0.604 & 0.612 & 0.487 & 0.538 & 0.636 & 0.559 & 0.542 \\
\hline x5-Fluorocytosine & 0.354 & 0.386 & 0.321 & 0.354 & 0.397 & 0.334 & 0.373 \\
\hline x5-Fluorouracil & 0.503 & 0.552 & 0.512 & 0.545 & 0.536 & 0.454 & 0.546 \\
\hline x6-Azauracil & 0.258 & 0.298 & 0.270 & 0.308 & 0.315 & 0.289 & 0.279 \\
\hline Xylose & 0.475 & 0.468 & 0.431 & 0.465 & 0.516 & 0.484 & 0.460 \\
\hline YNB & 0.508 & 0.541 & 0.481 & 0.519 & 0.543 & 0.411 & 0.525 \\
\hline YNB:ph3 & 0.151 & 0.18 & 0.144 & 0.195 & 0.194 & 0.153 & 0.166 \\
\hline YNB:ph8 & 0.295 & 0.345 & 0.315 & 0.356 & 0.354 & 0.267 & 0.334 \\
\hline
\end{tabular}


Table 1 continued

\begin{tabular}{llllllll}
\hline Trait/method & Bloom et al & Lasso & Ridge & BLUP & GBM & RF & SVM \\
\hline YPD & 0.533 & 0.546 & 0.480 & 0.515 & $\mathbf{0 . 5 5 6}$ & 0.469 & 0.524 \\
YPD:15C & $\mathbf{0 . 4 3 2}$ & 0.383 & 0.311 & 0.345 & 0.427 & 0.424 & 0.333 \\
YPD:37C & $\mathbf{0 . 7 1 1}$ & 0.653 & 0.576 & 0.606 & 0.691 & 0.686 & 0.603 \\
YPD:4C & 0.406 & 0.430 & 0.396 & 0.418 & $\mathbf{0 . 4 8 5}$ & 0.405 & 0.421 \\
Zeocin & 0.465 & 0.469 & 0.450 & 0.482 & $\mathbf{0 . 4 9 5}$ & 0.360 & 0.475 \\
Average rank & 4.37 & 3.14 & 6.27 & 3.74 & 1.66 & 4.96 & 3.86 \\
\hline
\end{tabular}

Table $2 \mathrm{cv} R^{2}$ for the five ML methods and for BLUP across 10 resampling runs applied to the wheat dataset. The best performance for each trait is in boldface. The average ranks for computation of the Friedman test are on the bottom line

\begin{tabular}{lllllll}
\hline Trait/method & Lasso & Ridge & BLUP & GBM & RF & SVM \\
\hline Yield (drought) & 0.023 & 0.060 & 0.217 & 0.051 & 0.172 & $\mathbf{0 . 2 1 9}$ \\
Yield (irrigated) & 0.084 & 0.162 & 0.253 & 0.132 & 0.184 & $\mathbf{0 . 2 5 8}$ \\
TKW & 0.172 & 0.240 & 0.277 & 0.218 & 0.242 & $\mathbf{0 . 3 0 4}$ \\
DTH & 0.292 & 0.325 & 0.381 & 0.325 & 0.358 & $\mathbf{0 . 3 9 4}$ \\
Average rank & 6.00 & 4.12 & 2.00 & 4.88 & 3.00 & 1.00 \\
\hline
\end{tabular}

Table $3 R^{2}$ for the five ML methods and for BLUP using a train-test split for the rice data. The best performance for each trait is in boldface. The average ranks for computation of the Friedman test are on the bottom line

\begin{tabular}{lllllll}
\hline Trait/method & Lasso & Ridge & BLUP & GBM & RF & SVM \\
\hline Culm diameter & 0.142 & $\mathbf{0 . 1 9 1}$ & 0.190 & 0.145 & 0.163 & 0.182 \\
Culm length & 0.529 & 0.567 & $\mathbf{0 . 5 6 8}$ & 0.559 & 0.539 & 0.516 \\
Culm number & 0.205 & 0.232 & 0.233 & 0.188 & 0.222 & $\mathbf{0 . 2 4 7}$ \\
Grain length & $\mathbf{0 . 3 8 7}$ & 0.375 & 0.381 & 0.371 & 0.361 & 0.383 \\
Grain width & 0.474 & 0.508 & $\mathbf{0 . 5 1 1}$ & 0.466 & 0.435 & 0.500 \\
Grain weight & 0.309 & 0.379 & 0.380 & 0.327 & 0.350 & $\mathbf{0 . 3 8 7}$ \\
Days to heading & 0.677 & 0.693 & $\mathbf{0 . 6 9 8}$ & 0.669 & 0.657 & 0.636 \\
Ligule length & 0.351 & 0.382 & 0.376 & 0.372 & 0.367 & $\mathbf{0 . 3 9 0}$ \\
Leaf length & 0.335 & 0.406 & 0.405 & 0.407 & 0.388 & $\mathbf{0 . 4 1 4}$ \\
Leaf width & 0.371 & 0.409 & 0.406 & 0.388 & 0.384 & $\mathbf{0 . 4 1 6}$ \\
Panicle length & 0.352 & 0.399 & 0.399 & 0.383 & 0.388 & $\mathbf{0 . 4 1 6}$ \\
Seedling height & 0.188 & 0.224 & $\mathbf{0 . 2 2 6}$ & 0.184 & 0.188 & 0.202 \\
Average rank & 4.88 & 2.38 & 2.12 & 4.50 & 4.71 & 2.42 \\
\hline
\end{tabular}

\subsection{Investigating the importance of mechanistic complexity}

The number of relevant attributes (markers, environmental factors), and the complexity of their interactions have an important impact on the ability to predict phenotype. We investigated how the mechanistic complexity of a phenotype impacted on the prediction results for the different prediction methods applied to the yeast dataset. Without a full mechanistic 


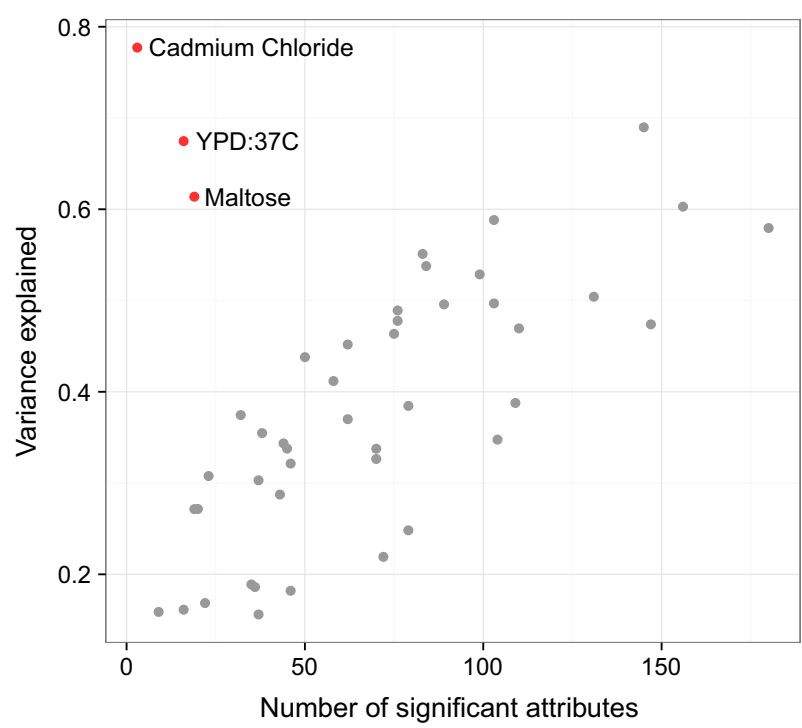

Fig. 4 Non-zero attributes selected by lasso regression in training sample plotted against variance explained in the test sample (yeast)

explanation for the cause of a phenotype, it is impossible to know the number of relevant attributes. However, in our yeast phenotype prediction data, which has no interacting environmental attributes, a reasonable proxy for the number of relevant attributes is the number of non-zero attributes selected by lasso regression (Fig. 4). This approach is not without caveats, as lasso might struggle with many small effects and, as previously mentioned, the choice between several highly correlated attributes is somewhat arbitrary.

To investigate the relationship between the number of markers chosen by the lasso and variance explained by the models we split the data into test (30\%) and training (70\%) sets, counted the number of non-zero parameters in the model fitted to the training set, and compared it to the model's performance on the test set. We observed that the environments with a higher proportion of variance explained tend to have a higher number of associated nonzero attributes. Notable exceptions to this are the three (cadmium chloride, YPD-37C, and maltose) in the top-left part of the graph, which has an unusually high $R^{2}$, but only a handful of associated non-zero attributes (only 6 markers for cadmium chloride). Notably, all three environments have a distinctive bimodal distribution that probably indicates that they are affected only by a few mutations.

We also wished to investigate how the complexity of the interactions of the attributes (in genetics the interaction of genes is termed 'epistasis', whilst interaction between alleles of the same gene is called 'dominance') affected relative performances of the models. Figure 5 shows pairwise plots of relative performances of the seven approaches with red circles corresponding to those traits for which Bloom identified pairs of interacting attributes, and blue triangles corresponding to those for which no interacting attributes were found. We observed that lasso outperforms or matches (difference of less than 0.5\%) Bloom's method on those traits where no interactions were detected. This is true for all 22 such traits. Furthermore, we observed that the lasso on the whole also slightly outperforms GBM for some traits, and considerably outperforms random forest for the traits with no interactions. For traits with identified interactions gradient boosting machines seem to show 


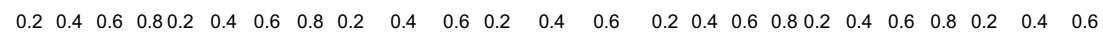
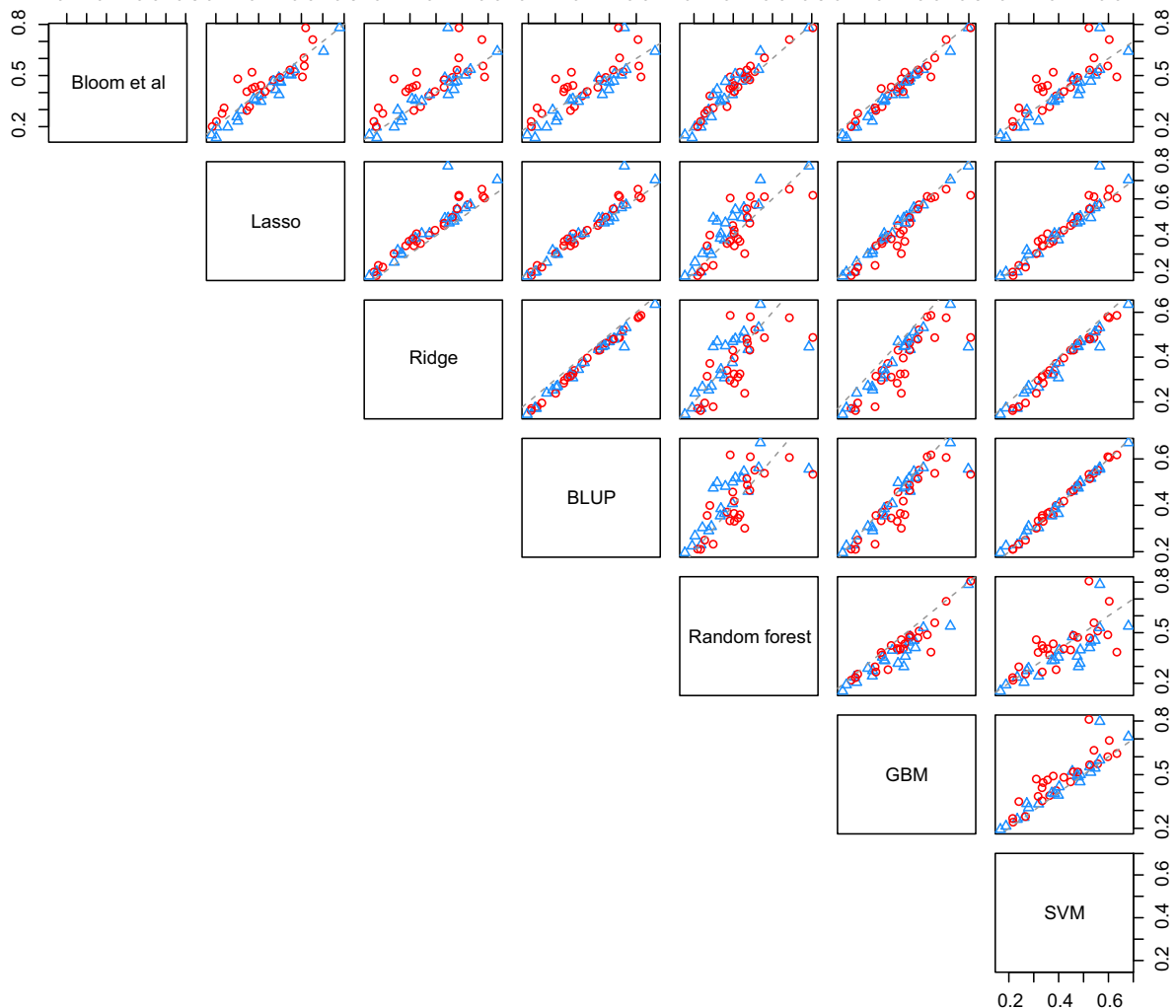

Fig. $5 \mathrm{cv} R^{2}$ for tenfold cross-validation for different ML models, genomic BLUP and method of Bloom et al. applied to the yeast data. Red circles and blue triangles correspond to traits with interactions and no interactions (as identified by Bloom et al.), respectively

the best performance relative to results of Bloom and random forest; the former outperforms GBM for only 6 traits out of 46 . Random forest seems to underperform compared to GBM and lasso, and it beats Bloom for only 14 traits with an average advantage of $1.9 \%$.

Finally, we noted optimal tuning parameters chosen for GBM in each CV fold for each trait. Scrutinising optimal tuning parameters might help us understand what makes a particular model suitable for a particular regression problem. In particular, we recorded tree interaction depth most frequently chosen across the 10 cross-validation folds (if two depths had equal frequency we took the smallest). Overall 1, 3 and 7 splits (corresponding to stumps, two- and three-way interaction trees, respectively) were chosen for 9, 18 and 19 traits, correspondingly. Looking closer, we noted that traits for which Bloom did not identify interacting attributes favoured stumps and shallow trees, while those with 2 or more interactions favoured deeper trees ( 7 splits $)$ - in fact, 7 splits were identified as the optimal tree depth for all traits with more than two interactions. We conclude that optimal GBM tree depth might help draw conclusions about the structure and complexity of the underlying data. 

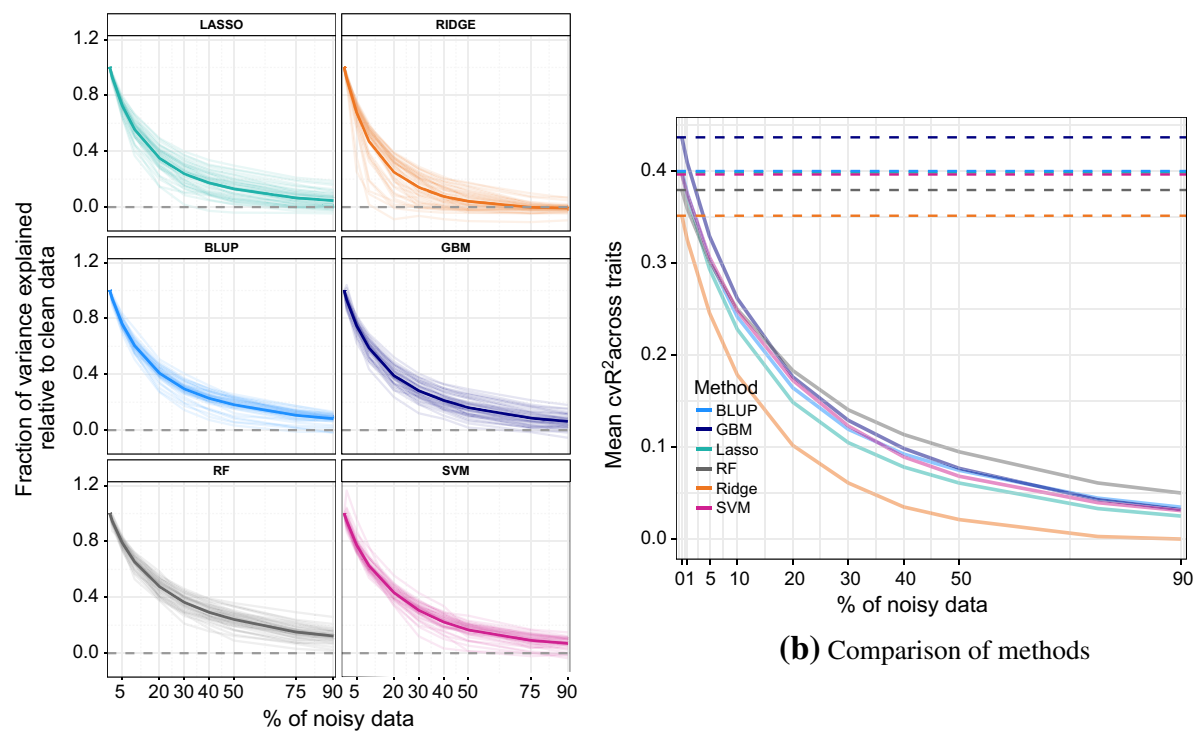

(b) Comparison of methods

(a)

Fig. 6 a Performance of ML and statistical methods under varying degrees of added class noise. The ratio of variance explained using noisy phenotype versus original data is plotted against proportion of noisy data added. The thick lines represent average value across all traits. b Comparison of methods on the absolute scale

\subsection{Investigating the importance of noise in the measured phenotype}

There is a great deal of noise in many real-world phenotype prediction problems. By noise, we mean both that the experimental conditions are not completely controlled, and the inherent stochastic nature of complex biological systems. Much of the factors affecting experimental conditions are environmental (e.g., soil and weather differences for crop phenotypes, different lifestyles in medical phenotypes), and this cannot be investigated using the yeast dataset. However, in many phenotype prediction problems, there is also a significant amount of class noise. To investigate the importance of such class noise, we randomly added or subtracted twice the standard deviation to a random subset of growth phenotype. We sequentially added noise to $5 \%, 10 \%, 20 \%, 30 \%, 40 \%, 50 \%, 75 \%$ and $90 \%$ of the phenotypic data for each trait and assessed performances of the statistical/machine learning methods on a test set (with training-testing split of 70\%-30\%). We repeated the procedure 10 times, each time selecting a different random subset of the data to add noise to. Figure 6a plots the ratio of variance explained using noisy phenotype versus original data versus proportion of noisy data added, averaged over the 10 runs. The results show a monotonic deterioration in accuracy with random forests performing the best, followed by GBM, BLUP, SVM, and lasso, with ridge regression trailing behind substantially. At about $20 \%$ of noisy data, RF starts to outperform GBM in terms of average $R^{2}$ (see Fig. 6b).

The algorithm underlying GBM is based on recursively explaining residuals of a model fit at the previous step, which might explain why it fares worse than RF under noise addition. For all the methods and traits, there seem to be very rapid deterioration in accuracy even for relatively small noise contamination (less than $10 \%$ of the data). 


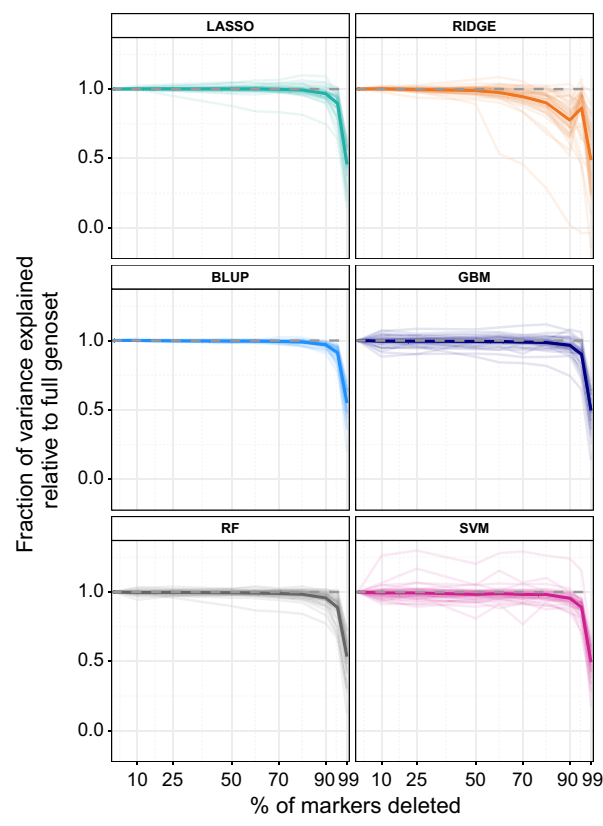

(a)

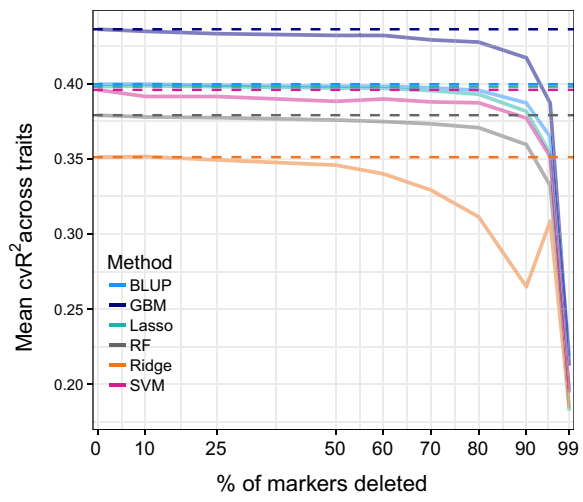

(b) Comparison of methods

Fig. 7 a Plots of the ratio of variance explained using a reduced marker set versus variance explained using the full marker set versus proportion of markers deleted for the five ML methods and BLUP. The thick lines represent average value across all traits. b Comparison of methods on the absolute scale

\subsection{Investigating the importance of number of genotypic attributes}

Another common form of noise in phenotype prediction studies is an insufficient number of markers to cover all sequence variations in the genome. This means that a genome is not fully represented, and other unobserved markers are present. To investigate this problem we sequentially deleted random $10 \%, 25 \%, 50 \%, 60 \%, 70 \%, 80 \%, 90 \%, 95 \%$ and $99 \%$ of the markers, and compared the performances of the five ML methods and BLUP on a test set (again with a training-testing split of 70\%-30\%). Figure 7a plots the ratio of variance explained using a reduced marker set versus variance explained using the full marker set versus proportion of markers deleted. Again these are average values over 10 runs with different random nested subsets of markers selected in each run. The statistical/machine learning methods, with the exception of ridge regression, lose minimal accuracy up until only $20 \%$ of the attributes remain, then undergo a rapid decline in accuracy after that. GBM and SVM seem to benefit from a reduced marker set for certain traits. BLUP's performance is very consistent across the traits and seems to be affected by marker deletion the least. Absolute performance across all traits of different methods relative to each other remains unchanged for all levels of marker deletion (see Fig. 7b).

The relative insensitivity to marker deletion across most traits and methods is not surprising given high linkage disequilibrium (correlation) between SNPs in the dataset (see Fig. 1a), which indicates high redundancy of markers. Indeed, we show in the next section that removal of correlated attributes hardly affects predictive performance of most methods for most traits. 
The steepest dropping line in plots for lasso, ridge, GBM, and RF corresponds to cadmium chloride; this is most likely because only a handful of important markers are likely to be governing the trait (see Fig. 4).

\subsection{Investigating the importance of data representation}

For each yeast strain, the marker data enabled us to recover the genomic structure formed by meiosis. We observed blocks of adjacent markers taking the same value, 1 or 0 (Fig. 1a). As yeast has been fully sequenced and annotated (Cherry et al. 2012), we also know where the markers are relative to the open reading frames (ORFs), continuous stretches of the genome which can potentially code for a protein or a peptide,- - 'genes'. Knowledge of this structure can be used to reduce the number of attributes with minimal loss of information. We then investigated two ways of doing this. In the first, we generated a new attribute for each gene (in which there are one or more markers) and assigned it a value of 1 if the majority of markers sitting in it had a value 1 , and 0 otherwise. In practice, we found that markers within each gene usually took on the same value for all but a handful of examples. Partially and fully overlapping genes were treated as separate. Markers within intergenic regions between adjacent genes were fused in a similar manner. Combining the gene and intergenic fused markers produced an alternative attribute set of 6064 binary markers.

The second way we investigated the fusing of blocks of markers was to group markers in genes and their flanking regions. To do this, we divided the genome into regions, each of which contained one gene together with half of the two regions between it and the two neighbouring genes. Partially overlapping genes were treated separately, but genes contained entirely within another gene were ignored. Markers lying within gene regions formed in this manner were fused according to the dominant value within this gene. This produced an alternative set of 4383 binary attributes.

We observed that the performance of the two alternative sets of genotypic attributes matched that of the full attribute set with the mean pairwise difference between any two attribute sets performance for each of the five ML methods, apart from ridge, not exceeding $0.5 \%$. Ridge regression's accuracy for some traits suffers considerably (e.g., cadmium chloride 5\%, maltose 5-12\%) when the reduced attribute sets were used. This indicates that most of the markers in blocks are in fact redundant as far as RF, GBM, SVM, and lasso are concerned.

\subsection{Investigating the importance of number of examples}

In phenotype prediction studies, there is often a shortage of data as it is expensive to collect. Traditionally obtaining the genotype data was most expensive, but increasingly data cost is being dominated by observation of the phenotype-with the cost of observing genotypes decreasing at super-exponential rate. To investigate the role of the number of examples, we successively deleted $10 \%, 25 \%, 50 \%, 60 \%, 70 \%, 80 \%$ and $85 \%$ of all sample points and assessed performance of the six statistical/ML models (again on a test set with a train-test split of $70 \%-30 \%$ and 10 resampling runs). Figure 8 a plots the ratio of variance explained using reduced dataset versus original data versus proportion of data deleted. Figure $8 \mathrm{~b}$ plots average performance over all traits for each method. The plots show that RF performed the most robustly. We note that by the time $50 \%$ of data is removed, RF is outperforming lasso, BLUP, and SVM, and at the $80 \%$ mark it starts outperforming GBM (in absolute terms across traits, see Fig. 8b). The original number of samples for each trait (which varied from 599 to 


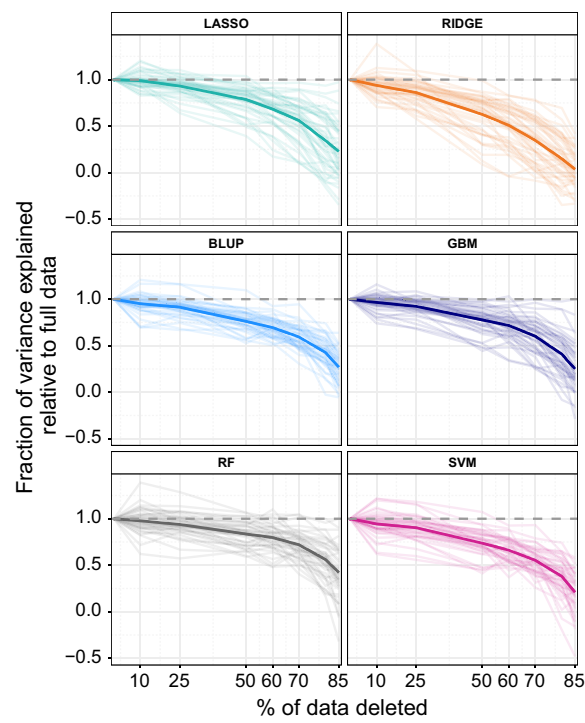

(a)

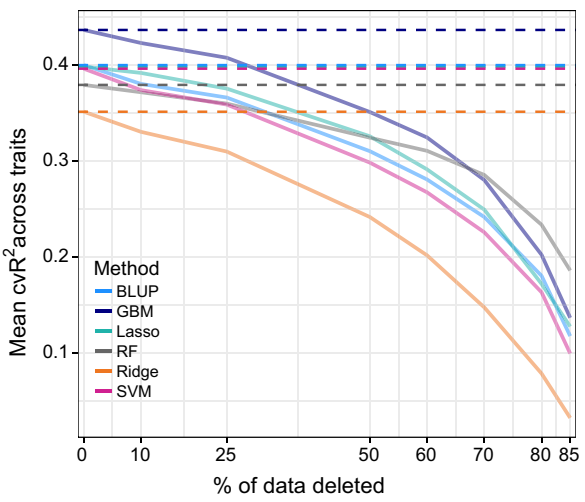

(b) Comparison of methods

Fig. 8 a Performance of ML methods and BLUP under sample points deletion. The ratio of variance explained using reduced dataset versus original is plotted against the proportion of data deleted. Thick lines represent average value across all traits. b Comparison of methods on the absolute scale

1007) did not seem to systematically affect the rate performance deterioration. We notice that there is more variation across the traits in response to sample point deletion, as compared to the addition of class noise and attribute deletion, where behaviour across the traits is more uniform (see Figs. 6, 7).

\subsection{Multi-task learning: learning across traits}

Rather than regarding the yeast dataset as 46 separate regression problems with 1008 sample points in each, in the spirit of multi-task learning one might consider it as a single large regression problem with $46 \times 1008$ observations (in practice less due to missing values). One would then hope that a predictive model will learn to differentiate between different traits, giving accurate predictions regardless of the environments. Moreover, letting a model learn from several traits simultaneously might enhance predictive accuracy for individual traits through drawing additional information from other (possibly related) traits. It is possible to consider this set-up also as a form of transfer learning (Caruana 1997; Evgeniou and Pontil 2004; Ando and Tong 2005). Most of the 46 traits are only weakly correlated (Pearson's correlation) but there are several clusters of phenotypes with much higher pairwise correlations. Hence, on top of considering a regression problem unifying all 46 traits, we also chose two smaller subsets of related traits with various levels of pairwise correlations:

(a) Lactate, lactose, sorbitol, and xylose: four sugar-related phenotypes with relatively high pairwise correlations (0.6-0.8).

(b) Lactate, lactose, sorbitol, xylose, ethanol, and raffinose: six sugar-related phenotypes with medium to high pairwise correlations $(0.42-0.8)$. 


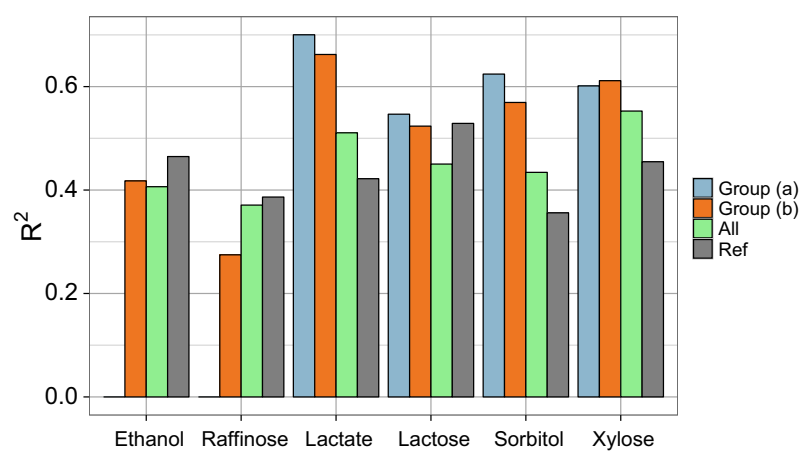

Fig. 9 RF prediction accuracy ( $R^{2}$ on a test set) for individual traits when trained on group (a) (blue bars), group $(b)$ (orange bars) and all of the 46 traits (green bars) compared to reference results (grey bars), when both learning and prediction was performed on individual traits

Grouping these traits makes sense given yeast biology: xylose, sorbitol, lactose, and raffinose are all sugars, and plausible environments for yeast to grow in; ethanol is a product of yeast sugar fermentation; while lactate participates in yeast metabolic process. Hence, it is not surprising that the six traits enjoy moderate to high pairwise correlations.

Combining several phenotype prediction problems into one results in each individual (yeast sample) having several entries in the input matrix and output vector-one for each trait. Additionally, we introduced an extra attribute, a categorical variable indicating which trait each sample corresponds to. We applied GBM and RF to this grouping approach. We show only random forest results, as GBM considerably underperformed compared to RF, and we did not apply SVM due to it being too computationally extensive for such a large problem. The performance was assessed by evaluation on a test set (30\% of the data), which was an aggregation of test sets of individual traits used throughout the paper; this made comparing the new results to reference results obtained by training on separate traits easier.

We assessed the performance of RF on the two groups of traits above as well as on the group comprising all 46 traits. The three models were assessed for overall prediction accuracy as well as for prediction accuracy for each trait. We compared the latter values to reference fits, models trained and tested on each trait separately. Figure 9 below shows the results. One can see that 3 out of 4 traits that belong to both groups $(a)$ and $(b)$ (lactate, sorbitol, xylose) benefited greatly from grouped learning (blue and orange bars) whilst predictive accuracy for the two additional traits in group $(b)$ (ethanol and raffinose) are substantially lower than reference. Moreover, adding these two traits reduced predictive accuracy for 3 out 4 of the original traits in group (a) (orange bars). Overall accuracy across all traits was $61 \%$ for group (a) and $52 \%$ for group $(b)$.

For any pair of traits in groups (a) and (b), the RF model trained on one of the traits has at least one of its top (as assessed by permutation OOB permutation tests) markers highly (or perfectly) correlated with one of the markers highlighted as important by RF model trained on the second trait. Likewise, RF models trained on groups (a) and (b) highlight highly (or perfectly) correlated markers. Models trained on combined traits, therefore, benefit from larger datasets to learn common genetic variants affecting these phenotypes.

For the full multi-task set-up using all 46 traits (green bars in Fig. 9), the overall accuracy of all traits was just $20 \%$. On average, predictive accuracy for individual traits for this model was $22 \%$ lower than reference results (models trained and tested just on one trait). The accuracy for cadmium chloride, for example, dropped to just $0.4 \%$. There were however 7 traits that 
benefited from grouped learning. Curiously, these included lactate, sorbitol, and xylose along with two other sugars trehalose and galactose (with an improvement of 5-10\%). However, the accuracies for lactate, sorbitol, and xylose were still lower than when these traits were considered as part of groups $(a)$ and $(b)$. It, therefore, seems that while combining multiple traits into a single regression problem indiscriminately might not, on the whole, improve overall or individual trait prediction accuracy, grouping carefully chosen traits with high pairwise correlation (perhaps advised by the organism's biology) can be advantageous.

\section{Discussion}

We have demonstrated the utility of the application of machine learning methods on three phenotype prediction problems. The results obtained compared favourably to those obtained from state-of-the-art classical statistical genetics methods.

The yeast problem investigated has the simplest form of phenotype prediction problem. The data is also as clean and complete as is currently possible, and this enabled us to gradually degrade the data in various ways to better understand the factors involved in prediction success and to make it resemble other types of phenotype prediction problem. In the original clean yeast data, GBM performed best, with lasso regression and the method of Bloom et al. joint second best.

The wheat and rice problems are typical of crop genome selection problems. For these datasets, SVM and BLUP were the best performing methods. We hypothesise that the success of BLUP is related to the population structure in these problems. Despite this success, BLUP does not optimally use population structure. Therefore, there is room to develop new machine learning methods that better use prior knowledge of population structure.

We investigated the role of the number of interactions between attributes. For the yeast dataset traits with no interactions, lasso proved to be the preferable method. We observed that GBM was the best method with traits with interacting attributes. In particular, traits with more than 2 interactions benefited deeper GBM trees. This is consistent with what would be expected by theory.

Out of the three types of noise we investigated, class noise seems to be by far the most damaging to the prediction accuracy of all the methods. This suggests that in collecting experimental data, greatest care should be taken in correctly recording class type. Of the machine learning methods, ridge regression's performance deteriorated the most under various forms of noise, while random forest was the most robust method.

An important observation inferred from applying the variety of classical and machine learning methods to three such different datasets, and to the modified yeast data, is that no method performs universally well. This is arguably a manifestation of the 'no free lunch theorem' of Wolpert and Macready (1997), which, simply speaking, asserts that no algorithm will perform better than any other when their performance is averaged over all possible problems.

Despite the no free lunch theorem, lessons can be learnt about which type of machine learning method suits what type of problem. GBM is shown to work very well on the clean yeast dataset. Here the lack of noise seems to suit the iterative fitting approach, which does not seem as robust as it could be in the presence of noise. RF is the most robust method in the presence of noise. This may be due to the bagging of prediction models, and it is possible that bagging could help other non-stable machine learning methods - though at the cost of additional computational overhead. SVM works well in both the real-world crop genetic 
problems. More work is required to identify which features of SVM are responsible for this success. More generally, determining which method performs better on which problem is a non-trivial problem and is the subject of meta-learning (e.g. Olier et al. (2018)).

One important form of phenotype prediction problem that we have not studied is human disease associations studies. These problems typically differ from the problems investigated here in several ways: there exists complex but unknown population structure, the environment of the examples is poorly controlled, and the phenotype investigated is a disease that may have a partly understood mechanism. Compared to the problems investigated here these properties make such problems in some ways easier, and some ways harder. We hypothesise that just as for the yeast, wheat, and rice datasets, the performance of off-the-shelf machine learning methods will compare favourably to those obtained from state-of-the-art classical statistical genetics methods.

There are a number of ways that machine learning methods could be developed for phenotype prediction problems. As mentioned in the introduction, the use of markers to describe genotypes is inefficient as it ignores their linear ordering, the type of sequence change, etc. One way to capture this information would be to use a relational representation (Getoor and Taskar 2007). The marker representation also ignores all the prior biological knowledge that is available about the genes involved. For a gene, there may be known tens/hundreds/thousands of facts. This knowledge is highly relevant to determining whether a gene is mechanistically involved, but is often ignored by both machine learning and classical statistical genetics methods. To include this knowledge in prediction, a good approach would perhaps be to use a hybrid relational/propositional approach that uses relational data mining to identify useful attributes (King et al. 2001). A relational approach could also explicitly include prior background knowledge about the population structure of the examples.

For some GWAS problems the goal is to produce new organisms with desired properties, and an example of this is plant breeding where the goal is to produce plants with increased crop yield, resistance to drought, etc. This suggests an application of active learning. But this would require the development of new active learning methods that take into account the specific way (meiosis) that new examples (organisms) are produced.

To conclude, there is relatively little communication between the machine learning and statistical genetics communities. This is unfortunate. Statistical genetics suffers from lack of access to new developments in machine learning, and machine learning suffers from a source of technically interesting, and societally important problems. We, therefore, hope that this paper will help bridge the differences between the communities and encourage machine learning research on phenotype prediction problems.

The original yeast data can be found at http://genomics-pubs.princeton.edu/YeastCross_ BYxRM/, wheat data at https://dl.sciencesocieties.org/publications/tpg/abstracts/5/3/103, and rice data at http://snp-seek.irri.org/_download.zul. Custom $\mathrm{R}$ code used to analyse the datasets can be found at: https://github.com/stas-g/grinberg-et-al-evaluation-of-ML-code.

Acknowledgements This work was supported by BBSRC Grant BB/J006955/1.

Open Access This article is distributed under the terms of the Creative Commons Attribution 4.0 International License (http://creativecommons.org/licenses/by/4.0/), which permits unrestricted use, distribution, and reproduction in any medium, provided you give appropriate credit to the original author(s) and the source, provide a link to the Creative Commons license, and indicate if changes were made. 


\section{References}

Alexandrov, N., Tai, S., Wang, W., Mansueto, L., Palis, K., Fuentes, R. R., et al. (2015). Snp-seek database of SNPs derived from 3000 rice genomes. Nucleic Acids Research, 43(D1), D1023-D1027.

Ando, R. K., \& Tong, Z. (2005). A framework for learning predictive structures from multiple tasks and unlabeled data. Journal of Machine Learning Research, 6, 1817-1853.

Armstead, I., Donnison, I., Aubry, S., Harper, J., Hörtensteiner, S., James, C., et al. (2007). Cross-species identification of Mendel's I locus. Science, 315(5808), 73.

Benjamini, Y., \& Hochberg, Y. (1995). Controlling the false discovery rate: A practical and powerful approach to multiple testing. Journal of the Royal Statistical Society: Series B, 57(1), 289-300.

Bloom, J. S., Ehrenreich, I. M., Loo, W. T., Lite, T.-L. V. o, \& Kruglyak, L. (2013). Finding the sources of missing heritability in a yeast cross. Nature, 494(7436), 234-237.

Brachi, B., Morris, G. P., \& Borevitz, J. O. (2011). Genome-wide association studies in plants: The missing heritability is in the field. Genome Biology, 12(10), 232.

Breiman, L. (2001). Random forests. Machine Learning, 45, 1-33.

Buckler, E. S., Holland, J. B., Bradbury, P. J., Acharya, C. B., Brown, P. J., et al. (2009). The genetic architecture of maize flowering time. Science, 325(5941), 714-718.

Caruana, R. (1997). Multitask learning. Machine Learning, 28, 41-75.

Casale, F. P., Rakitsch, B., Lippert, C., \& Stegle, O. (2015). Efficient set tests for the genetic analysis of correlated traits. Nature Methods, 12, 1-7.

Cherlin, S., Plant, D., Taylor, J. C., Colombo, M., Spiliopoulou, A., Tzanis, E., et al. (2018). Prediction of treatment response in rheumatoid arthritis patients using genome-wide snp data. Genetic Epidemiology, 42(8), 754-771.

Cherry, J. M., Hong, E. L., Amundsen, C., Balakrishnan, R., Binkley, G., et al. (2012). Saccharomyces genome database: The genomics resource of budding yeast. Nucleic Acids Research, 40(D1), D700-D705.

Clark, A. G. (2004). The role of haplotypes in candidate gene studies. Genetic Epidemiology, 27(4), 321-333.

Cortes, C., \& Vapnik, V. (1995). Support-vector networks. Machine Learning, 20(3), $273-297$.

de los Campos, G., Hickey, J. M., Pong-Wong, R., Daetwyler, H. D., \& Calus, M. P. L. (2013). Whole-genome regression and prediction methods applied to plant and animal breeding. Genetics, 193(2), 327-345.

De Los Campos, G., Naya, H., Gianola, D., Crossa, J., Legarra, A., Manfredi, E., et al. (2009). Predicting quantitative traits with regression models for dense molecular markers and pedigree. Genetics, 182(1), $375-385$.

Demšar, J. (2006). Statistical comparisons of classifiers over multiple data sets. Journal of Machine Learning Research, 7(Jan), 1-30.

Desta, Z. A., \& Ortiz, R. (2014). Genomic selection: Genome-wide prediction in plant improvement. Trends in Plant Science, 19(9), 592-601.

Dudoit, S., Fridlyand, J., \& Speed, T. P. (2002). Comparison of discrimination methods for the classification of tumors using gene expression data. Journal of the American Statistical Association, 97(457), 77-87.

Endelman, J. B. (2011). Ridge regression and other kernels for genomic selection with R package rrBLUP. The Plant Genome Journal, 4(3), 250.

Evgeniou, T., \& Pontil, M. (2004). Regularized multi-task learning. Proceedings of the 10th ACM SIGKDD International Conference on Knowledge Discovery and Data Mining, 109-117.

Friedman, J. (2001). Greedy function approximation: A gradient boosting machine. The Annals of Statistics, 29(5), 1189-1232.

Gamazon, E. R., Wheeler, H. E., Shah, K. P., Mozaffari, S. V., Aquino-Michaels, K., Carroll, R. J., et al. (2015). A gene-based association method for mapping traits using reference transcriptome data. Nature Genetics, 47(9), 1091.

Gauderman, J. W. (2002). Sample size requirements for matched case-control studies of gene-environment interaction. American Journal of Epidemiology, 155(5), 478-84.

Getoor, L., \& Taskar, B. (Eds.). (2007). Introduction to statistical relational learning, adaptive computation and machine learning. Cambridge: MIT Press.

Gianola, D., Fernando, R. L., \& Stella, A. (2006). Genomic-assisted prediction of genetic value with semiparametric procedures. Genetics, 173(3), 1761-1776.

Guan, Y., \& Stephens, M. (2011). Bayesian variable selection regression for genome-wide association studies and other large-scale problems. Annals of Applied Statistics, 5(3), 1780-1815.

Habier, D., Fernando, R. L., Kizilkaya, K., \& Garrick, D. J. (2011). Extension of the bayesian alphabet for genomic selection. BMC Bioinformatics, 12, 186.

Hayes, B., \& Goddard, M. (2010). Genome-wide association and genomic selection in animal breeding. Genome, 53(11), 876-883. 
Heffner, E. L., Sorrells, M. E., \& Jannink, J.-L. (2009). Genomic selection for crop improvement. Crop Science, 49(February), 1-12.

Hoerl, A. E., \& Kennard, R. W. (1970). Ridge regression: Biased estimation for nonorthogonal problems. Technometrics, 12(1), 55-67.

Hogben, L. T. (1946). An introduction to mathematical genetics. New York: W.W. Norton and Company.

Hsu, C.-W., Chang, C.-C., \& Lin, C.-J. (2008). A practical guide to support vector classification. BJU International, 101(1), 1396-400.

Jannink, J.-L., Lorenz, A. J., \& Iwata, H. (2010). Genomic selection in plant breeding: From theory to practice. Briefings in Functional Genomics, 9(2), 166-177.

Jaynes, E. T. (2003). Probability theory: The logic of science. Cambridge: Cambridge University Press.

Kang, H. M., Zaitlen, N. A., Wade, C. M., Kirby, A., Heckerman, D., Daly, M. J., et al. (2008). Efficient control of population structure in model organism association mapping. Genetics, 178(3), 1709-1723.

King, R. D., Srinivasan, A., \& Dehaspe, L. (2001). Warmr: A data mining tool for chemical data. Journal of Computer-Aided Molecular Design, 15(2), 173-181.

Korte, A., Vilhjálmsson, B. J., Segura, V., Platt, A., Long, Q., \& Nordborg, M. (2012). A mixed-model approach for genome-wide association studies of correlated traits in structured populations. Nature Genetics, 44(9), 1066-1071.

Lampa, E., Lind, L., Lind, P. M., \& Bornefalk-Hermansson, A. (2014). The identification of complex interactions in epidemiology and toxicology: A simulation study of boosted regression trees. Environmental Health, 13(1), 57.

Lee, S. H., \& van der Werf, J. (2016). MTG2: An efficient algorithm for multivariate linear mixed model analysis based on genomic information. Bioinformatics, 9(32), 1420-1422.

Lee, S. H., Wray, N. R., Goddard, M. E., \& Visscher, P. M. (2011). Estimating missing heritability for disease from genome-wide association studies. American Journal of Human Genetics, 88(3), 294-305.

Leung, M. K., Delong, A., Alipanahi, B., \& Frey, B. J. (2016). Machine learning in genomic medicine: A review of computational problems and data sets. Proceedings of the IEEE, 104(1), 176-197.

Li, J., Malley, J. D., Andrew, A. S., Karagas, M. R., \& Moore, J. H. (2016). Detecting gene-gene interactions using a permutation-based random forest method. BioData Mining, 9(1), 14.

Li, Z., \& Sillanpää, M. J. (2012). Overview of LASSO-related penalized regression methods for quantitative trait mapping and genomic selection. Theoretical and Applied Genetics, 125(3), 419-435.

Lin, Z., \& Altman, R. B. (2004). Finding haplotype tagging SNPs by use of principal components analysis. American Journal of Human Genetics, 75(5), 850-861.

Lippert, C., Listgarten, J., Liu, Y., Kadie, C. M., Davidson, R. I., \& Heckerman, D. (2011). FaST linear mixed models for genome-wide association studies. Nature Methods, 8(10), 833-835.

Liu, J., Peissig, P., Zhang, C., Burnside, E., McCarty, C., Page, D. (2012). Graphical-model based multiple testing under dependence, with applications to genome-wide association studies. The 28th Conference on Uncertainty in Artificial Intelligence (UAI) (pp. 511-522).

Locke, A. E., Kahali, B., Berndt, S. I., Justice, A. E., Pers, T. H., et al. (2015). Genetic studies of body mass index yield new insights for obesity biology. Nature, 518(7538), 197-206.

Loh, P.-R., Tucker, G., Bulik-Sullivan, B. K., Vilhjalmsson, B. J., Finucane, H. K., Salem, R. M., et al. (2015). Efficient bayesian mixed-model analysis increases association power in large cohorts. Nature Genetics, 47(3), 284.

Lynch, M., \& Walsh, B. (1998). Genetics and analysis of quantitative traits. Massachusetts: Sinauer Associates Inc.

Mackay, T. F. (2014). Epistasis and quantitative traits: Using model organisms to study gene-gene interactions. Nature Reviews Genetics, 15(1), 22-23.

Mansueto, L., Fuentes, R. R., Chebotarov, D., Borja, F. N., Detras, J., Abriol-Santos, J. M., et al. (2016). SNP-seek II: A resource for allele mining and analysis of big genomic data in oryza sativa. Current Plant Biology, 7, 16-25.

Marchini, J., Donnelly, P., \& Cardon, L. R. (2005). Genome-wide strategies for detecting multiple loci that influence complex diseases. Nature Genetics, 37(4), 413-417.

Meng, Z., Zaykin, D. V., Xu, C.-F., Wagner, M., \& Ehm, M. G. (2003). Selection of genetic markers for association analyses, using linkage disequilibrium and haplotypes. American Journal of Human Genetics, 73(1), 115-130.

Meuwissen, T. H. E., Hayes, B. J., \& Goddard, M. E. (2001). Prediction of total genetic value using genomewide dense marker maps. Genetics, 157(4), 1819-1829.

Mitchell, T. (1997). Machine learning. New York: McGraw-Hill.

Mittag, F., Büchel, F., Saad, M., Jahn, A., Schulte, C., Bochdanovits, Z., et al. (2012). Use of support vector machines for disease risk prediction in genome-wide association studies: Concerns and opportunities. Human Mutation, 33(12), 1708-1718. 
Ogutu, J. O., Piepho, H.-P., \& Schulz-Streeck, T. (2011). A comparison of random forests, boosting and support vector machines for genomic selection. BMC Proceedings, 5 Suppl 3(Suppl 3), S11.

Ogutu, J. O., Schulz-Streeck, T., \& Piepho, H.-P. (2012). Genomic selection using regularized linear regression models: Ridge regression, lasso, elastic net and their extensions. BMC Proceedings, 6(Suppl 2), S10.

Okser, S., Pahikkala, T., Airola, A., Salakoski, T., Ripatti, S., \& Aittokallio, T. (2014). Regularized machine learning in the genetic prediction of complex traits. PLoS Genetics, 10(11), e1004754.

Olier, I., Sadawi, N., Bickerton, G. R., Vanschoren, J., Grosan, C., Soldatova, L., et al. (2018). Meta-qsar: A large-scale application of meta-learning to drug design and discovery. Machine Learning, 107(1), 285-311.

Pirooznia, M., Seifuddin, F., Judy, J., Mahon, P. B., Potash, J. B., \& Zandi, P. P. (2012). Data mining approaches for genome-wide association of mood disorders. Psychiatric Genetics, 22(2), 55-61.

Poland, J., Endelman, J., Dawson, J., Rutkoski, J., Wu, S. Y., Manes, Y., et al. (2012). Genomic selection in wheat breeding using genotyping-by-sequencing. Plant Genome, 5(3), 103-113.

Price, A. L., Zaitlen, N. A., Reich, D., \& Patterson, N. (2010). New approaches to population stratification in genome-wide association studies. Nature Reviews, 11, 459-463.

Purcell, S., Neale, B., Todd-Brown, K., Thomas, L., Ferreira, M. A., Bender, D., et al. (2007). PLINK: A tool set for whole-genome association and population-based linkage analyses. The American Journal of Human Genetics, 81(3), 559-575.

R Core Team (2018). R: A language and environment for statistical computing.

Ray, S., \& Page, D. (2001). Multiple instance regression. ICML, 1, 425-432.

Rutkoski, J. E., Poland, J., Jannink, J.-L., \& Sorrells, M. E. (2013). Imputation of unordered markers and the impact on genomic selection accuracy. G3: Genes, Genomes, Genetics, 3(3), 427-439.

Schaid, D. J., Chen, W., \& Larson, N. B. (2018). From genome-wide associations to candidate causal variants by statistical fine-mapping. Nature Reviews Genetics, 19(8), 491-504.

Schizophrenia working group of the psychiatric genomics consortium. (2014). Biological insights from 108 schizophrenia-associated genetic loci. Nature, 511(7510), 421-427.

Shigemizu, D., Abe, T., Morizono, T., Johnson, T. A., Boroevich, K. A., Hirakawa, Y., et al. (2014). The construction of risk prediction models using GWAS data and its application to a type 2 diabetes prospective cohort. PLOS ONE, 9(3), e92549.

Speed, D., \& Balding, D. J. (2014). MultiBLUP: Improved SNP-based prediction for complex traits. Genome Research, 24(9), 1550-1557.

Spindel, J., Begum, H., Akdemir, D., Virk, P., Collard, B., Redoa, E., et al. (2015). Genomic selection and association mapping in rice (oryza sativa): Effect of trait genetic architecture, training population composition, marker number and statistical model on accuracy of rice genomic selection in elite, tropical rice breeding lines. PLOS Genetics, 11(2), 1-25.

Stranger, B. E., Stahl, E. A., \& Raj, T. (2011). Progress and promise of genome-wide association studies for human complex trait genetics. Genetics, 187(2), 367-383.

Sun, W., \& Tony Cai, T. (2009). Large-scale multiple testing under dependence. Journal of the Royal Statistical Society. Series B. Statistical Methodology, 71(2), 393-424.

Szymczak, S., Biernacka, J. M., Cordell, H. J., Gonzalez-Recio, O., König, I. R., Zhang, H., et al. (2009). Machine learning in genome-wide association studies. Genetic Epidemiology, 33(S1), S51-S57.

Tibshirani, R. (1996). Regression shrinkage and selection via the lasso. Journal of the Royal Statistical Society: Series B, 58(1), 267-288.

VanRaden, P. M. (2008). Efficient methods to compute genomic predictions. Journal of Dairy Science, 91, 4414-4423.

Wakefield, J. (2007). A Bayesian measure of the probability of false discovery in molecular genetic epidemiology studies. American Journal of Human Genetics, 81(2), 208-227.

Wang, S., \& Zhao, H. (2003). Sample size needed to detect gene-gene interactions using association designs. American Journal of Epidemiology, 158(9), 899-914.

Wei, W.-H., Hemani, G., \& Haley, C. S. (2014). Detecting epistasis in human complex traits. Nature Reviews Genetics, 15(11), 722-733.

Westfall, P. H., Zaykin, D. V., \& Young, S. S. (2002). Multiple tests for genetic effects in association studies. In S. W. Looney (Ed.), Methods in molecular biology (Vol. 184, pp. 143-168). New York: Humana Press.

Widmer, C., Lippert, C., Weissbrod, O., Fusi, N., Kadie, C., Davidson, R., et al. (2014). Further improvements to linear mixed models for genome-wide association studies. Scientific Reports, 4, 6874.

Wolpert, D. H., \& Macready, W. G. (1997). No free lunch theorems for optimization. Transactions on Evolutionary Computation, 1(1), 67-82.

Wood, A. R., Esko, T., Yang, J., Vedantam, S., Pers, T. H., Gustafsson, S., et al. (2014). Defining the role of common variation in the genomic and biological architecture of adult human height. Nature Genetics, 46(11), 1173. 
Wray, N. R., Yang, J., Hayes, B. J., Price, A. L., Goddard, M. E., \& Visscher, P. M. (2013). Pitfalls of predicting complex traits from SNPs. Nature Reviews Genetics, 14(7), 507-515.

Wright, M. N., Ziegler, A., \& König, I. R. (2016). Do little interactions get lost in dark random forests? BMC Bioinformatics, 17(1), 145.

Yang, J., Benyamin, B., McEvoy, B. P., Gordon, S., Henders, A. K., Nyholt, D. R., et al. (2010). Common SNPs explain a large proportion of the heritability for human height. Nature Genetics, 42(7), 565-569.

Zhang, Z., Ersoz, E., Lai, C.-Q., Todhunter, R. J., Tiwari, H. K., Gore, M. A., et al. (2010). Mixed linear model approach adapted for genome-wide association studies. Nature Genetics, 42, 355-360.

Zhou, X., Carbonetto, P., \& Stephens, M. (2013). Polygenic modeling with bayesian sparse linear mixed models. PLoS Genetics, 9(2), e1003264.

Zhou, X., \& Stephens, M. (2014). Efficient multivariate linear mixed model algorithms for genome-wide association studies. Nature Methods, 11(4), 407-409.

Ziegler, A., DeStefano, A. L., König, I. R., Bardel, C., Brinza, D., et al. (2007). Data mining, neural nets, trees-problems 2 and 3 of genetic analysis workshop 15. Genetic Epidemiology, 31 Suppl 1(S1), S51-S60.

Zou, H., \& Hastie, T. (2005). Regularization and variable selection via the elastic net. Journal of the Royal Statistical Society: Series B Statistical Methodology, 67(2), 301-320.

Publisher's Note Springer Nature remains neutral with regard to jurisdictional claims in published maps and institutional affiliations.

\section{Affiliations}

\section{Nastasiya F. Grinberg ${ }^{1,2}(\mathbb{D}) \cdot$ Oghenejokpeme I. Orhobor $^{1} \cdot$ Ross D. King $^{3}$}

Oghenejokpeme I. Orhobor

oghenejokpeme.orhobor@manchester.ac.uk

Ross D. King

rossk@chalmers.se

1 School of Computer Science, University of Manchester, Oxford Road, Manchester M13 9PL, UK

2 Present Address: Department of Medicine, Cambridge Institute of Therapeutic Immunology \& Infectious Disease, Jeffrey Cheah Biomedical Centre, Cambridge Biomedical Campus, University of Cambridge, Cambridge CB2 0AW, UK

3 Department of Biology and Biological Engineering, Division of Systems and Synthetic Biology, Chalmers University of Technology, Kemivägen 10, SE-412 96 Gothenburg, Sweden 